\author{
S. Colagiuri · K. Borch-Johnsen - C. Glümer • \\ D. Vistisen
}

\title{
There really is an epidemic of type 2 diabetes
}

Received: 22 December 2004 / Accepted: 17 May 2005 / Published online: 9 July 2005

(C) Springer-Verlag 2005

\begin{abstract}
Abbreviations DETECT-2: Evaluation of Screening and Early Detection Strategies for Type 2 Diabetes and Impaired Glucose Tolerance study · WHO: World Health Organization
\end{abstract}

The number of people with diabetes has increased throughout the world, and the rate of increase shows no signs of slowing. The Diabetes Atlas estimates that there were 194 million people with diabetes in 2003 and predicts an increase to 333 million by 2025 [1]. Figures from the World Health Organization (WHO) are similar [2]. These prediction models do not require an increase in the incidence of diabetes, but anticipate that the total number of individuals with diabetes will increase because of improved life expectancy, population growth and progressive urbanisation.

Studies from many parts of the world have reported an increasing age-specific prevalence of diabetes. Recent data from the National Health and Nutrition Examination Survey (NHANES) studies in the USA show that the prevalence of diagnosed diabetes is relatively constant within each stratum of BMI, but imply that an increasing prevalence and incidence of diabetes would be expected as a consequence of increasing obesity [3]. In Denmark, three

\author{
S. Colagiuri \\ Department of Endocrinology and Diabetes, \\ Prince of Wales Hospital, \\ Sydney, NSW, Australia \\ K. Borch-Johnsen $(\bowtie) \cdot C$. Glümer $\cdot$ D. Vistisen \\ Steno Diabetes Center, \\ Niels Steensensvej 2, \\ 2820 Gentofte, Denmark \\ e-mail: kbjo@steno.dk \\ Tel.: +45-4443-9415 \\ Fax: $+45-4443-8232$ \\ K. Borch-Johnsen \\ Faculty of Health Science, University of Aarhus, \\ Aarhus, Denmark
}

population-based surveys were carried out (in 1974, 1996 and 2000) in 60-year-old men and women living in the same geographical area in greater Copenhagen. These showed that prevalence increased from 7.8 through 12.3 to $14.0 \%$ in men, and from 5.6 through 6.8 to $13.6 \%$ in women $[4,5]$. Although this might suggest an increasing incidence of diabetes, it could also be explained by other factors, including the longer survival of individuals with a diagnosis of diabetes. Thus, the central question is: to what extent can this increasing prevalence be explained by improved life expectancy and demographic factors, as against a simple increase in incidence or an imbalance between incidence and mortality? The last of these explanations was first put forward by Støvring et al. [6], using data from a Danish pharmacoepidemiological database, and has been developed further by Green et al. in this issue of the journal [7].

The term 'diabetes epidemic' has been used in recent years to describe the increasing burden of this disease. The term 'epidemic' was first coined in relation to infectious diseases and refers to a substantial increase in the number of new cases over a short, defined period of time [8]. More recently, the term has been extended to non-communicable diseases such as diabetes and to risk factors such as obesity; however, no specific, universally agreed definition has been adopted. Although the term has been used to describe an increasing prevalence, a true 'epidemic' would require an increasing incidence of diabetes. In consequence, loose terminology is partly responsible for the current dilemma. We will discuss potential explanations for the increasing prevalence of diabetes and will use different data sets to quantify the influence of each of these alternative explanations within a model system.

\section{How to explain the increasing prevalence of diabetes}

This issue is not only of academic importance; it also has important implications for the allocation of limited re- 


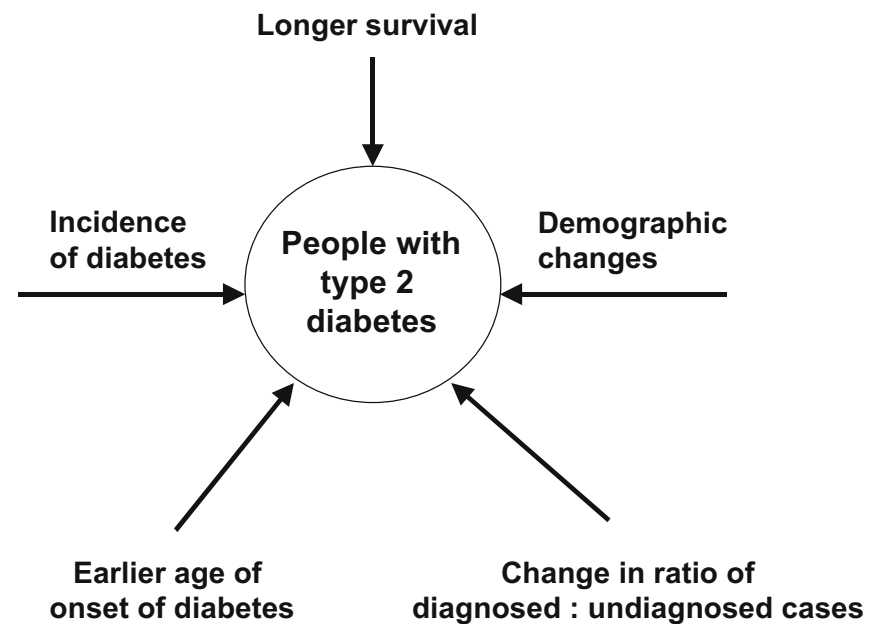

Fig. 1 Diabetes epidemiological model. Factors directly affecting the prevalence of diabetes included in the present analysis

sources for diabetes care and prevention. The changing prevalence of diabetes is based upon point estimates in a dynamic environment. It is essential to determine and explore the components of this environment before we address the question of whether or not there is an 'epidemic'.

Figure 1 illustrates the model used in this paper to address this question. As depicted, five factors directly affect the prevalence of diabetes: (1) the ratio of diagnosed : undiagnosed cases of diabetes; (2) population demographic changes; (3) age at onset of diabetes; (4) mortality in patients with diabetes and in the general population; and (5) incidence of diabetes. In addition to these five factors, an imbalance between incidence and mortality, induced by an increasing incidence in the past, will also lead to a nonsteady-state situation and thus, as discussed below, to an increasing prevalence of diabetes.

We have tested these potential explanations in two models, one reflecting an industrialised Western society and one reflecting a developing region. Each of the two models is based on theoretical populations of 1,000,000 inhabitants aged 30-59 years, because the age range was restricted in the Finnish data. The age and sex distribution in each region is based on population data from the US Census Bureau [9], which contains demographic information from all regions. The 'Western' model is based on data from two different population-based surveys performed in Finland $[10,11]$, while the model for the developing region is based on two population-based surveys from Samoa [12; personal communication, S. Viali, Samoa Ministry of Health and Oceania University of Medicine Samoa]. For both areas, mortality data for the non-diabetic population were provided by the WHO [13]. Based on these surveys, the prevalence of diabetes was estimated at two time points, which were separated by a 5-year interval. Table 1 shows baseline data for the two populations. For the Westernised region, the prevalence increased from 2.5 to $4.7 \%$ over a period of 5 years, which translates into a relative increase in prevalence of $88 \%$. For the developing region, the corresponding increase was from 11.7 to $17.9 \%$, equivalent to a $53 \%$ increase in prevalence. The following analysis examines the extent to which this increase in prevalence can be explained by each of the five factors mentioned above.

\section{Ratio of diagnosed : undiagnosed cases of diabetes}

Increased diagnostic activity leading to an increasing ratio of diagnosed : undiagnosed diabetes might generate a spurious increase in the prevalence of known diabetes. However, data from the Evaluation of Screening and Early Detection Strategies for Type 2 Diabetes and Impaired Glucose Tolerance study (DETECT-2) database [14] indicate that the ratio of diagnosed : undiagnosed cases may actually be decreasing, which would have the effect of underestimating any true change in the prevalence of known diabetes. Since the present analysis is based on populationbased surveys that included all diagnosed and undiagnosed cases of diabetes, it is not affected by changes in the ratio of diagnosed : undiagnosed cases.
Table 1 Age distribution for each of the two populations used in the model

For each region the model was based on a population of $1,000,000$. The age distributions for the Westernised and developing regions are based on the age structures for Finland and Samoa, as provided by the US Census Bureau, and the diabetes prevalence rates are based on the data from two cross-sectional prevalence studies conducted in Finland $[10,11]$ and Samoa [12, personal communication, S. Viali, Samoan Ministry of Health and Oceania University of Medicine, Samoa], respectively

\begin{tabular}{|c|c|c|c|c|c|}
\hline & \multirow[t]{2}{*}{ Age } & \multicolumn{2}{|c|}{ Proportion of the population (\%) } & \multicolumn{2}{|c|}{ Prevalence of diabetes $(\%)$} \\
\hline & & Time $=0$ & Time $=5$ years & Time $=0$ & Time $=5$ years \\
\hline \multirow[t]{7}{*}{ Westernised region } & $30-34$ & 21 & 18 & 0.6 & 1.3 \\
\hline & $35-39$ & 22 & 19 & 1.0 & 2.1 \\
\hline & $40-44$ & 16 & 20 & 1.7 & 3.4 \\
\hline & $45-49$ & 14 & 17 & 2.8 & 5.3 \\
\hline & $50-54$ & 13 & 14 & 3.9 & 7.3 \\
\hline & $55-59$ & 14 & 12 & 6.8 & 12.1 \\
\hline & Total & 100 & 100 & 2.5 & 4.7 \\
\hline \multirow[t]{7}{*}{ Developing region } & $30-34$ & 26 & 26 & 0.9 & 7.7 \\
\hline & $35-39$ & 20 & 21 & 9.2 & 14.0 \\
\hline & $40-44$ & 16 & 17 & 11.5 & 17.1 \\
\hline & $45-49$ & 14 & 14 & 16.5 & 23.6 \\
\hline & $50-54$ & 13 & 12 & 20.9 & 28.4 \\
\hline & $55-59$ & 11 & 10 & 25.8 & 33.1 \\
\hline & Total & 100 & 100 & 11.7 & 17.9 \\
\hline
\end{tabular}


Changes in population demographics

The population of most countries is ageing, with a consequent increase in the proportion that are middle aged or elderly. Since the prevalence of diabetes increases with age, these demographic changes will lead to an increase in the prevalence of diabetes in the entire population and, thus, to an increasing number of individuals with diabetes.

Table 1 shows the age distribution at baseline and 5 years later in the hypothetical population of 1,000,000 in each of the two regions (the age range for both regions was set at $30-59$ years, and mean ages of 43 years and 42 years were used for the Westernised region and the developing region, respectively). As shown in Table 1, ageing of the population only affects those in the 40-54-year-old age group in Western societies and those in the 35-49-year-old age group in the developing countries, while the relative contribution of the oldest age groups decreases. As a consequence of this, the total effect on the crude prevalence of diabetes in the 30-59-year-old age group is minimal. In the Westernised region, the observed increase in prevalence was $2.2 \%$, of which $0.005 \%$ could be explained by demographic changes. In the developing region, changing demography did not contribute to the increase in prevalence.

\section{Decreasing age at onset of diabetes}

The increasing age-specific prevalence of diabetes, particularly in the younger age groups $[1,2]$, suggests that the age at onset of diabetes may be decreasing. In the absence of true incidence studies, the magnitude of this change is unknown. We, therefore, used the age of diagnosis of screen-detected diabetes as a proxy for age of onset. We estimated the change in age at onset based on the age distribution among previously undiagnosed cases of diabetes reported by the repeated population-based surveys from Finland, India, the Pacific Islands and North America included in the DETECT-2 database. According to our estimation, the age at diagnosis decreased by 0.06 years to 0.35 years per calendar year. Thus, for the present analysis, we modelled decreases in age of onset of 0,1 and 2 years over a 5-year period. A decrease in age at onset of 1 year would explain an increase in prevalence of $0.3 \%$, as against the $2.2 \%$ observed in the Westernised region, and would explain $0.6 \%$ of the $6.2 \%$ increase in the developing region. If age at onset decreased by 2 years, this would explain an increase in prevalence of $0.6 \%$ in the Westernised region and $1.1 \%$ in the developing region.

\section{Decreasing mortality}

Unfortunately, there is little information available concerning mortality over time in the diabetic population. We have used the mortality rates for the general population reported by WHO [12] as an indicator of mortality in the non-diabetic population. The mortality of the diabetic population was then estimated using the hazard ratio provided by the

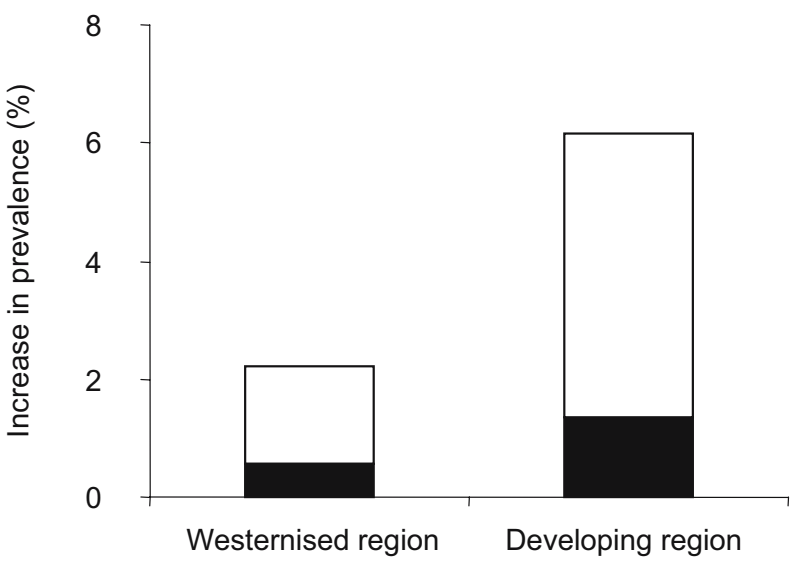

Fig. 2 Five-year increase in the prevalence of diabetes in the Westernised region (modelled based on Finland) and the developing region (modelled based on surveys from Samoa). The dark area represents the fraction of the 5-year increase that could be directly attributed to changes in age at onset, population demographic changes and decreasing excess mortality in people with diabetes

Diabetes Epidemiology: Collaborative Analysis of Diagnostic Criteria in Europe (DECODE) study [15] for the Westernised region and from the Diabetes Epidemiology: Collaborative Analysis of Diagnostic Criteria in Asia (DECODA) study [16] for the developing region. We then modelled a decrease of between 10 and $25 \%$ in the excess mortality from all causes in people with diabetes. These reflect an optimistic estimate based on the most successful intervention studies in type 2 diabetic patients [17] and are greater than the recently reported reduction in excess mortality found in the USA [18]. Even so, the most optimistic scenario would only explain $0.02 \%$ of the total $2.2 \%$ increase in prevalence in the Westernised region and $0.2 \%$ of the $6.2 \%$ increase observed in the developing region.

The combined effect of all the above-mentioned factors - demography, age at onset and mortality - could explain, at best, a $0.6 \%$ increase in prevalence, corresponding to $26.0 \%$ of the total increase of $2.2 \%$ observed over the 5 years in the Westernised region. In the developing region, these factors would explain a $1.4 \%$ increase in prevalence, corresponding to $22.0 \%$ of the observed increase of $6.2 \%$ in this region (Fig. 2). In other words, $70-80 \%$ of the increase in prevalence is unexplained and must be due to factors other than those listed above.

\section{How can the remaining increase in prevalence be explained?}

Although an increasing incidence of type 2 diabetes (gradual or rapid, current or recent) might account for the unexplained increase in prevalence, this is not the only possibility. A steady incidence rate with decreasing mortality would also result in an increasing prevalence of diabetes [6]. The ideal way to distinguish between these possibilities would be by means of long-term, well-designed incidence studies unbiased by changes in detection rates. Unfortunately, such studies are not available. Studies 
such as the one by Støvring et al. [6] have used prescription of drugs for diabetes as a proxy variable for incidence. This has the potential for bias; first because of possible changes in detection rates, and second because of possible changes in prescription practices over the study period, given that only drug-treated patients were included. Consequently, this imprecise estimate of incidence is highly dependent on treatment policies and temporal changes in prescription strategies.

As an alternative approach, we have estimated the incidence rates needed to reach the 5-year increases calculated in the present study. This was achieved by applying the mortality rates used previously in our model and by assuming that mortality and incidence rates remained constant over the 5-year period. Based on this assumption, an annual incidence of diabetes of 0.6 per 100 person-years in the Westernised region and 2.5 per 100 person-years in the developing region would be needed to explain the increase in prevalence. These estimates are high relative to the reported incidence rates in non-Hispanic white individuals in the San Antonio Heart Study (0.5-0.6 per 100 personyears) [19] and in a study conducted in Mauritius (2.0-2.5 per 100 person-years) [20]. These were both cohort studies of populations that were $2-3$ years older at entry than the populations modelled in our study, and were followed for 5-8 years. Thus, at the end of follow-up, the cohorts were 7-11 years older than the populations included in our model. These differences in age structure, in combination with the higher than reported incidence rates required to explain the increased prevalence of diabetes in our model, suggest a previous or ongoing increase in incidence in both regions, even though decreasing mortality will also contribute to the increasing prevalence of diabetes. Although a Danish study [6] and data from Mauritius [20] both suggest a relatively constant incidence, the confidence intervals provided would allow for a relative increase in incidence of $3 \%$ per year, which is consistent with our calculations.

\section{Conclusions}

An increasing prevalence of diabetes has been seen in most populations throughout the world, but the explanation is less straightforward than might be expected given a dynamic environment with improving health status, increased longevity and global migration. Multiple factors, therefore, influence the overall prevalence of diabetes and total burden of disease.

We modelled the influence of five potential factors on the prevalence of diabetes in a Westernised region and in a developing region over a 5 -year period. We found that improved life expectancy, decreasing age of onset and population demographic changes could explain no more than $20-25 \%$ of the total increase in the prevalence of diabetes, even using assumptions favouring the hypothesis that these factors can explain the increasing prevalence. A long-term constant imbalance between incidence and mortality would also contribute markedly, but is unlikely to provide a full explanation for the difference. Taking this into account, incidence rates of $0.6 \%$ per year in the Westernised region and $2.5 \%$ per year in the developing region were needed to explain the observed increase in prevalence. These estimates are relatively high compared with reported figures, suggesting that a true increase in incidence (ongoing or recent) is needed to explain the observed increase in prevalence. This is consistent with reports from the USA that the prevalence of diabetes is constant within BMI strata, but with a marked shift of BMI towards higher levels, a shift that would intuitively be expected to cause an increasing incidence of diabetes.

There is no doubt that both the total number of diabetic individuals worldwide and the total prevalence of diabetes in adults will continue to increase as a consequence of changing population demography, improved treatment and decreasing mortality. Although this might not represent an epidemic according to the strict definitions used in the epidemiological literature, many will consider the term appropriate in relation to the increasing global burden of diabetes.

\section{References}

1. International Diabetes Federation (2003) Diabetes atlas. 2 edn. International Diabetes Federation, Brussels

2. Wild S, Roglic G, Green A, Sicree R, King H (2004) Global prevalence of diabetes: estimates for the year 2000 and projections for 2030. Diabetes Care 27:1047-1053

3. Gregg EW, Cadwell BL, Cheng YJ et al (2004) Trends in the prevalence and ratio of diagnosed to undiagnosed diabetes according to obesity levels in the U.S. Diabetes Care 27:28062812

4. Drivsholm T, Ibsen H, Schroll M, Davidsen M, Borch-Johnsen K (2001) Increasing prevalence of diabetes mellitus and impaired glucose tolerance among 60-year-old Danes. Diabetic Med 18:126-132

5. Glumer C, Jorgensen T, Borch-Johnsen K (2003) Prevalences of diabetes and impaired glucose regulation in a Danish population: the Inter99 study. Diabetes Care 26:2335-2340

6. Støvring H, Andersen M, Beck-Nielsen H, Green A, Vach W (2003) Rising prevalence of diabetes: evidence from a Danish pharmaco-epidemiological database. Lancet 362:537-538

7. Green A, Støvring H, Andersen M, Beck-Nielsen H (2005) The epidemic of type 2 diabetes is a statistical artefact. Diabetologia DOI 10.1007/s00125-005-1841-0

8. Last JM (2001) A dictionary of epidemiology. 4th edn. Oxford University Press, New York

9. US Census Bureau (http://www.census.gov/ipc/www/idbprint. html), Table 094, last accessed: 26 November 2004

10. Vartiainen E, Jousilahti P, Alfthan G, Sundvall J, Pietinen P, Puska P (2000) Cardiovascular risk factor changes in Finland, 1972-1997. Int J Epidemiol 29:49-56

11. WHO MONICA Project Principal Investigators (1988) The World Health Organization MONICA Project (monitoring trends and determinants and in cardiovascular disease): a major international collaboration. J Clin Epidemiol 41:105-114

12. Collins VR, Dowse GK, Toelupe PM et al (1994) Increasing prevalence of NIDDM in the Pacific island population of Western Samoa over a 13-year period. Diabetes Care 17:288296

13. Mortality Web-page, WHO (http://www3.who.int/whosis/menu. cfm?path=whosis, life) last accessed 16 November 2004

14. Borch-Johnsen K, Colagiuri S, Balkau B et al (2004) Creating a pandemic of prediabetes: the proposed new diagnostic criteria for impaired fasting glycaemia. Diabetologia 47:1396-1402 
15. The DECODE study group on behalf of the European Diabetes Epidemiology Group (1999) Glucose tolerance and mortality: comparison of WHO and American Diabetes Association diagnostic criteria. Lancet 354:617-621

16. Nakagami T, DECODA Study Group (2004) Hyperglycaemia and mortality from all causes and from cardiovascular disease in five populations of Asian origin. Diabetologia 47:385-394

17. Gaede P, Vedel P, Larsen N, Jensen GVH, Parving HH, Pedersen O (2003) Multifactorial intervention and cardiovascular disease in patients with type 2 diabetes. N Engl J Med 348:383-393
18. McBean AM, Li S, Gilbertson DT, Collins AJ (2004) Differences in diabetes prevalence, incidence, and mortality among the elderly of four racial/ethnic groups: whites, blacks, Hispanics, and Asians. Diabetes Care 27:2317-2324

19. Haffner SM, Miettinen H, Stern MP (1997) Are risk factors for conversion to NIDDM similar in high and low risk populations? Diabetologia 40:62-66

20. Soderberg S, Zimmet P, Tuomilehto J et al (2004) High incidence of type 2 diabetes and increasing conversion rates from impaired fasting glucose and impaired glucose tolerance to diabetes in Mauritius. J Intern Med 256:37-47 